\title{
THE EXAMINATION OF THE FAMILY TREES OF PATIENTS DIAGNOSED WITH GESTATIONAL DIABETES MELLITUS WITH REGARD TO DIABETES
}

\author{
Ayşegül ALIOOĞULLARI*, Yeliz DOĞAN MERİH*, Dilek COŞKUNER POTUR**, \\ Çiğdem SEZER* \\ * Zeynep Kamil Obstetrics and Pediatrics Research and Training Hospital \\ ${ }^{* *}$ Marmara University Faculty of Health Sciences Department of Nursing
}

Problem statement: Pregnant women with a history of diabetes mellitus in their families are in the risk group for gestational diabetes mellitus

Purpose: This study was planned in order to examine the first degree and close relatives of pregnant women who were diagnosed with gestational diabetes mellitus, and to evaluate their predilection for gestational diabetes mellitus.

Method: The study was conducted between the months of July and December 2016 in a Obstetrics and Pediatrics Research and Training Hospital in the city of Istanbul with the participation of 91 women who presented to the diabetes nurse for counseling, were diagnosed with gestational diabetes mellitus, and agreed to participate in the study. Data was collected through the completion of the questionnaires developed by the researchers via face to face interviews, and this data was then evaluated. The SPSS 21.0 program was used in the evaluation of research results.

Results: The mean age of the 91 pregnant women in the context of the study who were diagnosed with gestational diabetes mellitus was $32.41 \pm 4,88$, while their mean weight was $79,80 \pm 13,31$ and their mean weight gain during pregnancy was $8.20 \mathrm{~kg}$. Among the 91 patients who were not diagnosed with chronic diabetes $23.1 \%$ were primipara, and $34.1 \%$ of those who were multipara were found to be diagnosed with gestational diabetes mellitus in their previous pregnancies. $26.4 \%$ of the women were found to use tobacco before pregnancy, $11 \%$ were found to continue smoking, $47.3 \%$ to have a diet list for gestational diabetes, $37.4 \%$ were found to eat according to their diets, and $63.7 \%$ were found to dominantly eat fruit and vegetables. $93.4 \%$ of the pregnant women were found to regularly visit for check ups during pregnancy. When the presence of chronic diseases before pregnancy were checked, it was found that $78 \%$ had no chronic disease, $13.2 \%$ had thyroid related chronic diseases, $8.8 \%$ had other chronic diseases such as anemia and high blood pressure, and that $16.5 \%$ had a medicine they regularly used.
Among the pregnant women who were diagnosed with gestational diabetes, $35.2 \%$ had diabetes in their mother, $36.3 \%$ in their father, $9.9 \%$ in their sister, and $7.7 \%$ in their brother. When the status of pregnant women with gestational diabetes with regard to the presence of diabetes in their relatives from their mother's side was examined, the rate of having the disease in their relatives was found to be $51.6 \%$. Among those, $16.5 \%$ had diabetes in their aunt, $15.4 \%$ in their uncle, $20.9 \%$ in their grandmother, and $8.8 \%$ in their grandfather. When the status of pregnant women with gestational diabetes with regard to the presence of diabetes in their relatives from their father's side was examined, the rate of having the disease in their relatives was found to be $58.2 \%$. Among those, $27.5 \%$ had diabetes in their aunt, $16.5 \%$ in their uncle, $19.8 \%$ in their grandmother, and $7.7 \%$ in their grandfather. Only $17.58 \%$ of the women didn't have a diagnosis of diabetes in their relatives.

Conclusions: When pregnant women with gestational diabetes were examined with regard to a diagnosis of diabetes in their first degree and close relatives, pregnant women with diabetes in their father's side were found to be higher in number. Thus, such patients can be suggested to be monitored closely for gestational diabetes mellitus from the start of pregnancy

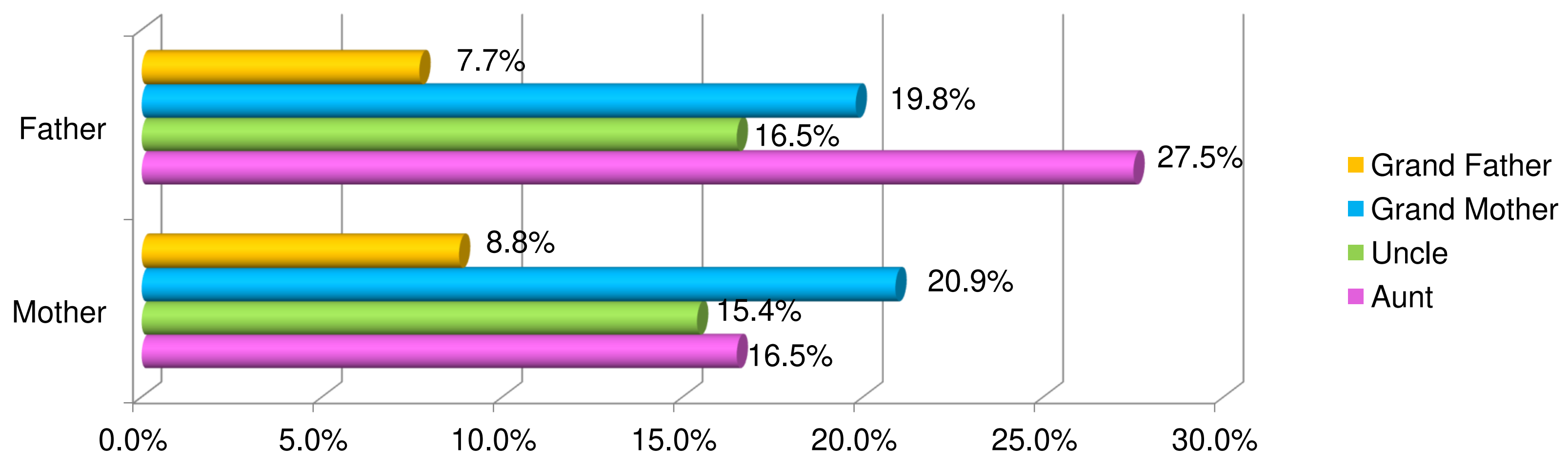

